\title{
Diffractive Structure Functions from the H1 and ZEUS Experiments at HERA
}

\author{
Irina A. Korzhavina ${ }^{\mathrm{a}, *}$ \\ ${ }^{a}$ Experimental High Energy Department, Skobeltsyn Institute of Nuclear Physics, Moscow State University, \\ Vorobjevy Gory 1/2, GSP-1, 119991 Moscow, Russia.
}

\begin{abstract}
The cross section of inclusive diffractive process $e p \rightarrow e X p$ was measured within a wide kinematic range in the H1 and ZEUS experiments at HERA. Results obtained by different experiments and methods are compatible within measurement uncertainties. The measurements were subjected to DGLAP next-to-leading order QCD global fits and the diffractive parton distribution functions (DPDFs) of the proton were determined with noticeably reduced uncertainties due to very high precision of the data. The gluon density precision was much improved in fits which also included data on dijet production in diffractive DIS. Predictions based on the determined DPDFs are in agreement with the measured inclusive cross-section of diffractive dijet photoproduction and charm production in diffractive DIS at HERA. The longitudinal diffractive structure function $F_{L}^{D}$ was measured directly for the first time.
\end{abstract}

Keywords: diffraction, factorisation, QCD fits, structure function, parton distribution function, HERA, H1, ZEUS

\section{Introduction}

Studies of the dissociation of virtual photons, $\gamma^{*} p \rightarrow$ $X p$, in diffractive deep inelastic ep scattering (DIS), $e p \rightarrow e X p$, at HERA contributed significantly to the understanding the dynamics of diffraction in terms of quarks and gluons and to further development of perturbative Quantum Chromodynamics (pQCD) calculation techniques [1]. These processes constitute a large fraction $(\sim 10 \%)$ of the visible DIS cross section and are mostly originating from the hard scattering of partons. Theoretically predicted factorisation [2] was confirmed in measurements of diffractive dijet production by HERA experiments $\mathrm{H} 1$ and ZEUS. In studies of diffractive scattering it was used for QCD fits to measure momentum distributions of partons in the proton, called diffractive parton distribution functions (DPDFs).

The differential diffractive DIS cross section is measured in the kinematic variables of the exchanged boson virtuality $Q^{2}$, the inelasticity $y$, the fraction of the momentum of the proton carried by the diffractive exchange $x_{P}$, the Bjorken variable defined for the diffractive exchange $\beta$, and the four-momentum transfer squared at the proton vertex $|t|$.

\footnotetext{
${ }^{*}$ On Behalf of the $\mathrm{H} 1$ and ZEUS Collaborations

Email address: irina@mail.desy.de (Irina A. Korzhavina)
}

The cross section may be presented in terms of the diffractive reduced cross section $\sigma_{r}^{D(4)}$ related to the diffractive structure functions, $F_{2 / L}^{D(4)}$, as:

$$
\begin{aligned}
& \sigma_{r}^{D(4)}\left(\beta, Q^{2}, x_{P}, t\right)=\frac{\beta Q^{4}}{2 \pi \alpha^{2} Y_{+}} \frac{d^{4} \sigma(e p \rightarrow e X p)}{d \beta d Q^{2} d x_{P} d t} \\
& =F_{2}^{D(4)}\left(\beta, Q^{2}, x_{P}, t\right)-\frac{y^{2}}{Y_{+}} F_{L}^{D(4)}\left(\beta, Q^{2}, x_{P}, t\right),
\end{aligned}
$$

where $Y_{+}=1+(1-y)^{2}$. Like the inclusive structure functions, the $F_{2 / L}^{D(4)}$ are defined being the proton structure functions, probed in a process with a fast proton of fractional momentum $\left(1-x_{P}\right)$ in the final state.

In accord to the factorisation theorem, diffractive cross section can also be presented as a sum of hard scattering cross sections for a parton $i$ convoluted with DPDFs $f_{i}^{D}$. Thus the diffractive structure functions, $F_{2 / L}^{D(4)}$ are related to the DPDFs through the convolutions with coefficient functions $C_{2 / L, i}$ :

$F_{2 / L}^{D(4)}\left(\beta, Q^{2}, x_{P}, t\right)=\sum_{i} \int_{\beta}^{1} \frac{d z}{z} C_{2 / L, i}\left(\frac{\beta}{z}\right) f_{i}^{D}\left(z, x_{P} ; Q^{2}, t\right)$, where $\mathrm{z}$ is the longitudinal momentum fraction of the parton entering the hard subprocess with respect to the diffractive exchange. In the lowest order sub-process $z=\beta$. The inclusion of higher order sub-processes leads to $\beta<z$.

\section{Diffractive Cross Section Measurements}

At high centre-of-mass energy, diffractive $e p$ scattering has two specific signatures. A leading proton carrying 
most (> 90\%) of the beam energy may be directly detected (proton tagging measurement) in the final state using Proton Spectrometers (PS: H1 (V)FPS or ZEUS LPS). There may also be identified a final state with no hadronic activity in a large rapidity region, called Large Rapidity Gap (LRG). The LRG separates outgoing (untagged) proton (or (untagged) low mass hadronic system $Y$ originating from proton dissociation) from the rest of the hadronic system $X$.

Lately H1 [3] and ZEUS [4] collaborations measured reduced cross sections of the diffractive process $e p \rightarrow e X Y$ with improved precision compared to earlier measurements, both the PS and LRG measurement techniques being used. Though the ranges accessed by the $\mathrm{H} 1$ and ZEUS measurements do not quite coincide with each other (Fig. 1) both data lie within the kinematic limits: $2<Q^{2}<700 \mathrm{GeV}^{2},|t|<1.0 \mathrm{GeV}^{2}, x_{P}<0.1$, $M_{Y}(P S)=m_{p}$ or $M_{Y}(L R G)<1.6 \mathrm{GeV}$ and full range of $\beta$. There is a global normalisation difference at the $\sim 10-13 \%$ level between the measurements of the two experiments (Fig. 1 up) compatible with uncertainties.

To match $\mathrm{H} 1$ and ZEUS cross sections the ZEUS data were scaled by the factors consistent with unity accounting for the normalisation uncertainties (Fig. 1 down). Apart from the normalisation discrepancy, H1 and ZEUS high precision measurements show good agreement in shapes of distributions throughout most of the phase space studied no matter what a measurement method used.

To test the compatibility between the results from the different measurement techniques and to estimate the proton dissociation admixture to the LRG data, the ratio of the $\sigma_{r}^{D(3)}$ values obtained with the PS method to those obtained with the LRG one was studied (Fig. 2). No subtraction of the proton-dissociative admixture to the LRG data was performed. Within experimental uncertainties the ratio is independent of $x_{P}, Q^{2}$ or $\beta$. The normalisation difference is ascribed to the proton dissociative contribution which typically amounts 20\% of the LRG sample as estimated in both experiments. $\sigma_{r}^{D(3)}$ measurements with the PS and the LRG methods are consistent in the region of overlap.

\section{Diffractive parton distribution functions}

The latest high precision set of DPDFs [4] was determined in the global QCD fits to ZEUS LRG and LPS cross section data [5] combined with data on diffractive dijet production in DIS [6]. Only data with $Q^{2}>5$ $\mathrm{GeV}^{2}$ could be fitted within the combined framework of DGLAP evolution and proton vertex factorisation. The
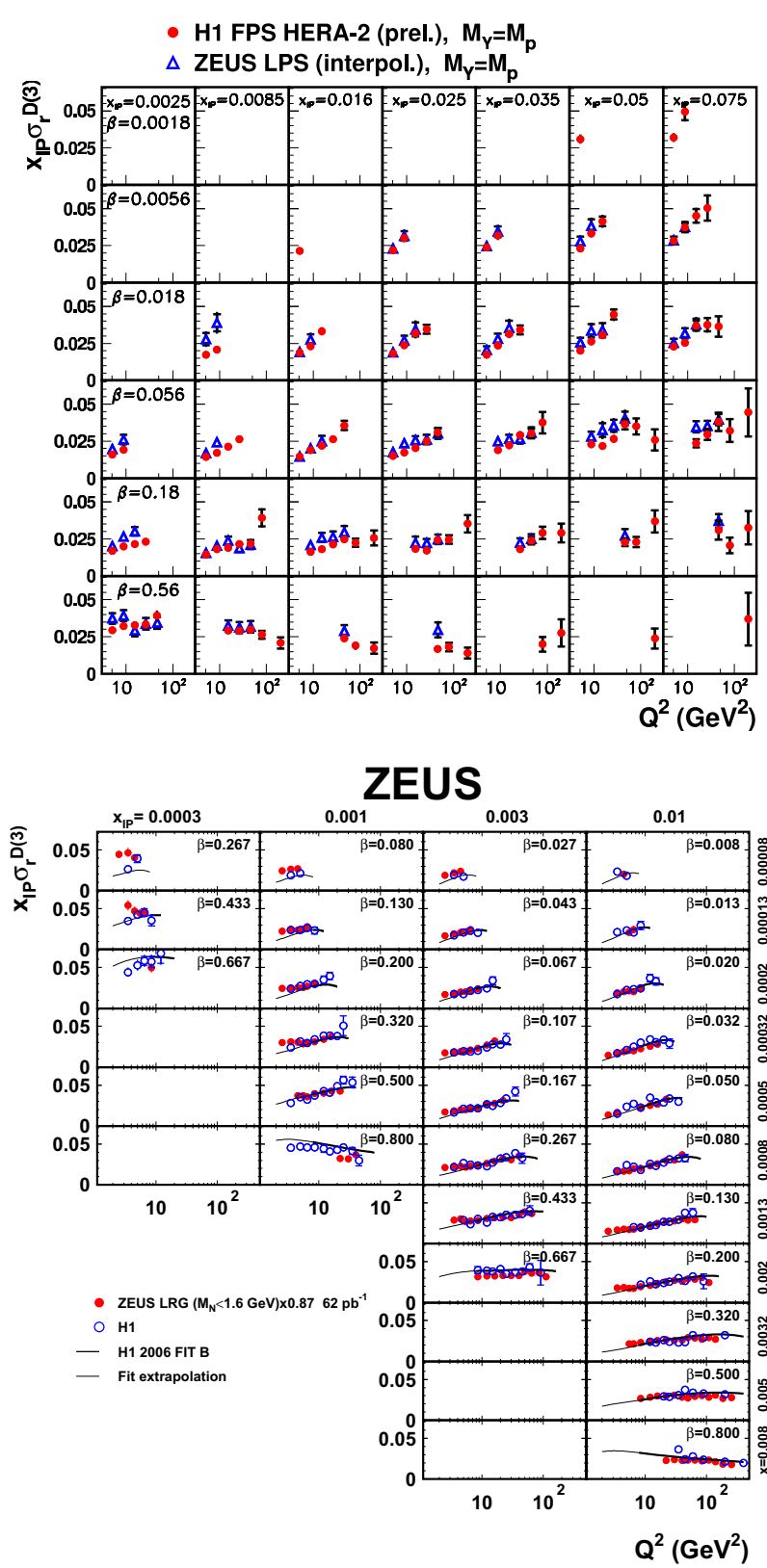

Figure 1: Comparison between the $\mathrm{H} 1$ and ZEUS measurements of the diffractive reduced cross section $\sigma_{r}^{D(3)}$ as a function of $Q^{2}$ in $\left(x_{P}, \beta\right)$ bins, PS (up) or LRG (down) data being used.

extracted DPDFs correspond to the single-diffractive reaction with a proton in the final state and are valid in the region $|t|<1 \mathrm{GeV}^{2}, M_{X}>2 \mathrm{GeV}, x_{P}<0.1$. The conventional DGLAP formalism in next-to-leading order (NLO) of QCD was applied. The parton distributi- 

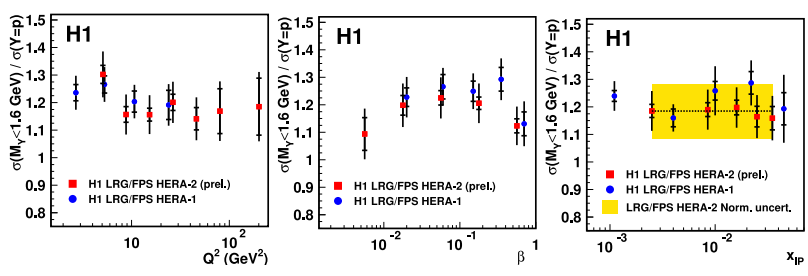

ZEUS

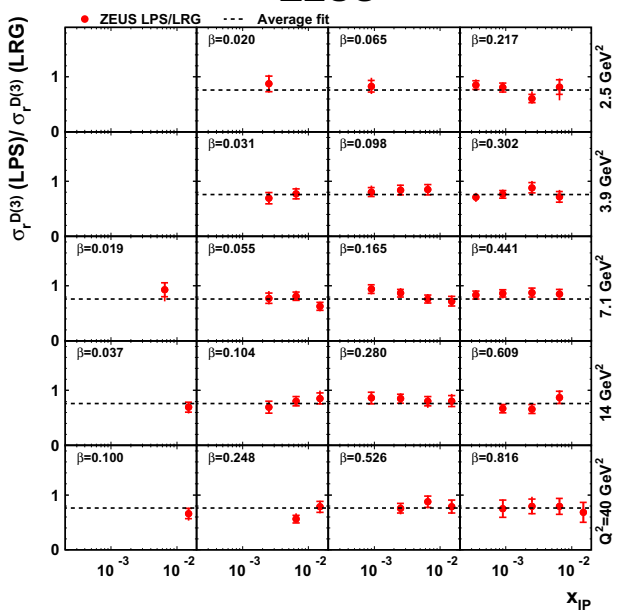

Figure 2: The ratio of the reduced diffractive cross sections, $\sigma_{r}^{D(3)}$, obtained with the LPS and the LRG methods, as functions of $Q^{2}, \beta$ or $x_{P}$ (up, H1) or a function of $x_{P}$ for different $\left(Q^{2}, \beta\right)$ bins (down, ZEUS). The proton dissociative background was not subtracted from the LRG data. The lines indicate the average value of the ratio.

ons at the starting scale $\left(Q_{0}^{2}\right)$ were parameterised with polynomials in $z: A z^{B}(1-z)^{C}$. There were tried two fits (S and $\mathrm{C}$ ) to the inclusive data and a fit (SJ) to the combined inclusive and dijet data. Parameterisations of the gluon density at the starting scale were taken to be very different to estimate theoretical uncertainties. Fits $\mathrm{S}$ and $\mathrm{SJ}$ were based on the parameterisation with $A_{g}, B_{g}$ and $C_{g}$ parameters free. In fit $\mathrm{C}$ the gluon density was taken to be a constant: $A_{g}=$ const, $B_{g}=0$ and $C_{g}=0$.

The resulting quark and gluon densities are presented in Fig. 3. All fits (fit $\mathrm{S}$ is not shown) gave similar distributions (upper plots) with small uncertainties for quark densities. Fits $\mathrm{S}$ and $\mathrm{C}$ gave very different distributions for the gluon density, particularly at high $z$. Fit $\mathrm{S}$ grows rapidly with $z \rightarrow 1$, while fit $C$ vanishes as $z \rightarrow 1$ in a smooth way. The gluon density from fit SJ (down plots) is similar to that of fit $\mathrm{C}$ and fit $\mathrm{S}$ is ruled out. Diffractive dijet data are able to discriminate between different gluon parameterisations due to dominance of boson-gluon fusion mechanism in the production of jets. Combined fits to inclusive and dijet data constrain both the quark and gluon DPDFs to a good comparable pre- cision across the whole $\mathrm{z}$ range.

According to QCD factorisation, the DPDFs measured should be able to describe cross sections for other diffractive processes: charm production in deep inelastic scattering [9] and jet photoproduction [10]. Predictions based on the fit ZEUS DPDF SJ for the charm contribution to the diffractive structure function were found in fair agreement with the data (plots not shown) for $x_{P}$ of 0.004 and 0.02 and $Q^{2}$ of 4 and $25 \mathrm{GeV}^{2}$. The predic-

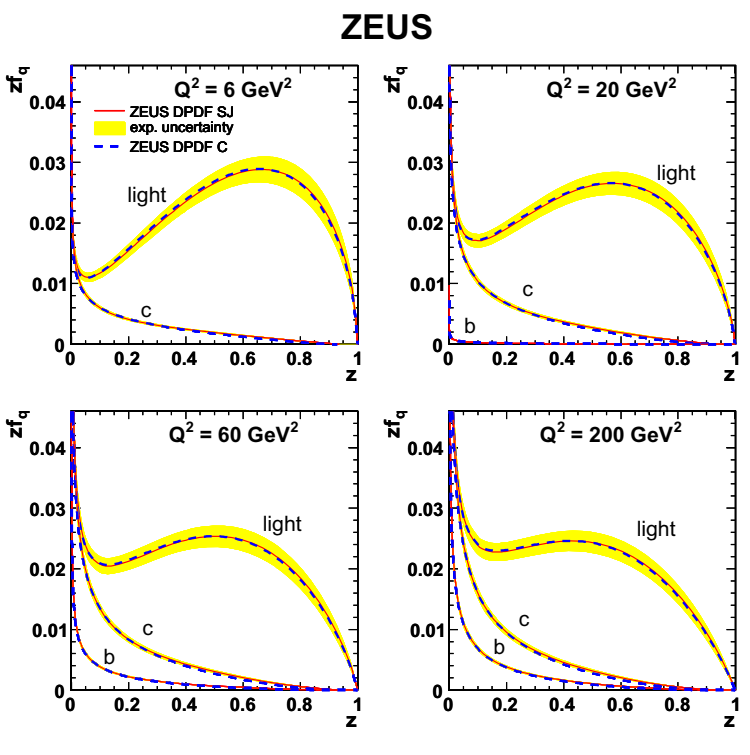

ZEUS
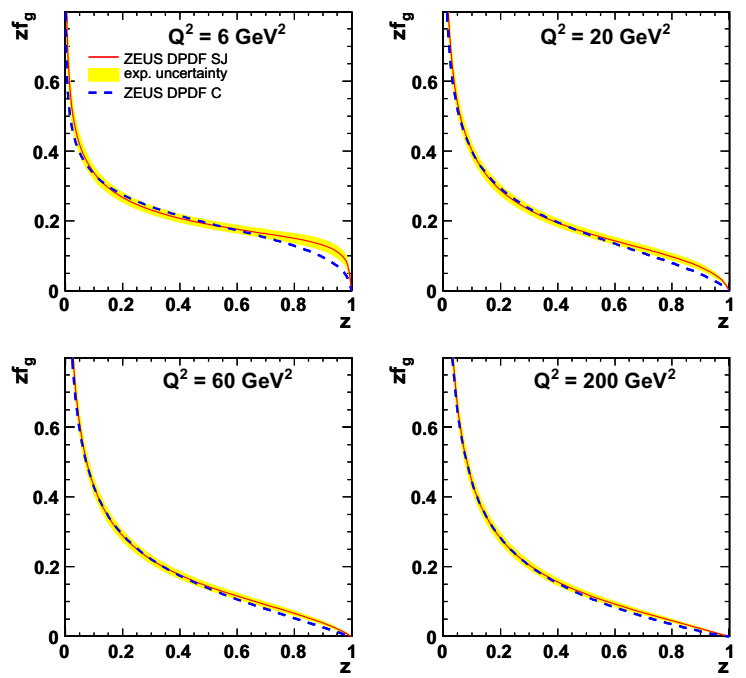

Figure 3: Quark (up) and gluon (down) distributions from $\sigma_{r}^{D}\left(Q^{2}\right)$ measurements with LRG, LPS and dijet ZEUS data. Shown are the total experimental uncertainties only. The starting scale was $Q_{0}^{2}=$ $1.8 \mathrm{GeV}^{2}$ with strong coupling constant, $\alpha_{s}\left(M_{Z}\right)=0.118$ and heavy quark masses $m_{c}=1.35 \mathrm{GeV}, m_{b}=4.3 \mathrm{GeV}$ were used. 
tions based on the fit ZEUS DPDF SJ for the diffractive dijet photoproduction cross sections in $x_{\gamma}^{o b s}$, the fraction of the photon energy participated in producing the dijet system, and in the transverse energy $E_{T}^{j e t}$ of the leading jet agree with the data over the whole $x_{\gamma}^{o b s}$ and $E_{T}^{\text {jet }}$ ranges (plots not shown). No suppression either of the resolved photon component, or of both components globally is observed.

\section{Measurement of $F_{L}^{D}$}

The DIS diffractive reduced cross section at low $Q^{2}$ is determined by structure functions $F_{2}^{D}$ and $F_{L}^{D}$ in a linear form (see sec. 1). Thus measurements of $\sigma_{r}^{D}$ in fixed $\left(x, Q^{2}\right)$ bins at different $y$ allow to measure $F_{2}^{D}$ and $F_{L}^{D}$ simultaneously and in model independent manner. From linear fits to $\sigma_{r}^{D}$ in $y / Y_{+}$one obtains $F_{2}^{D}$ as an intercept with the cross section axis and $F_{L}^{D}$ being the slope of the fit. The precision of the fit depends on the range available in $y$. Whilst $F_{2}^{D}$ is determined by the sum of weighted quark and anti-quark distributions only, $F_{L}^{D}$ additionally includes gluon contribution which at low $x$ exceeds quark ones. Therefore $F_{L}^{D}$ is a direct measure of the gluon distribution at low $x$. Such measurement can be used for testing other methods.

As $Q^{2}=$ sxy different values of $y$ can be reached by variation in $s . F_{L}^{D}\left(x, Q^{2}\right)$ was measured for the first time by the $\mathrm{H} 1$ experiment [7] in the range $2.5<Q^{2}<7$ $\mathrm{GeV}^{2}$ and $0.001<x_{P}<0.01$ at proton beam energies 460,575 and $920 \mathrm{GeV}$. Within the experimental uncertainties the preliminary result of the measurements (Fig. 4). is consistent with the predictions based on $\mathrm{H} 1$ 2006 DPDF [8] Fit A and Fit B extrapolated to low $Q^{2}$, i.e. being in good agreement with the NLO QCD picture of diffraction.

\section{Conclusions}

The full HERA data sample has been analysed to measure diffractive parton density functions to the best precision possible. As it is desirable to combine all available diffractive DIS data sets into an unique HERA set, $\mathrm{H} 1$ and ZEUS measurements by various methods with very different systematics have been compared in detail to test the control over the systematics and agreement between the different measurements.

All measurements are found to be consistent in the shapes of the distributions throughout most of the phase space. Compatible estimations of the proton dissociation contributions in the LRG samples were obtained. There was found a global normalisation difference at the $13 \%$ level between the similar measurements of the two

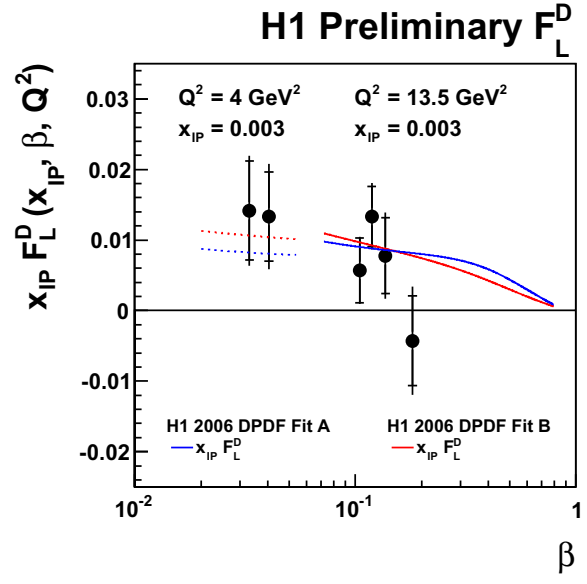

Figure 4: $x_{P} F_{L}^{D}$ as a function of $\beta$ measured at $Q^{2}=4 \mathrm{GeV}^{2}$ and $Q^{2}=13.5 \mathrm{GeV}^{2}$ (earlier result). Predictions from NLO QCD fits H1 2006 Fit A (blue line) and Fit B (red line) were estimated with starting scale $Q^{2}=8.5 \mathrm{GeV}^{2}$ and extrapolated to $Q^{2}=4 \mathrm{GeV}^{2}$ (dotted lines)

experiments. The data are well described by the QCD fit and the quark densities (from the inclusive data) and gluon densities (from the dijet data) are constrained to similar precision. The first $F_{L}^{D}$ measurement is in agreement with DPDF prediction.

Since $\mathrm{H} 1$ and ZEUS diffractive data are not yet combined, further improvement in accuracy of DPDFs is possible.

\section{Acknowledgements}

It is a pleasure to thank DESY for providing me with financial support.

\section{References}

[1] HERA and the LHC, Proceedings of the 2nd, 3rd and 4th HERA-LHC Workshops, eds. de Roeck, Jung (2009); http://www.desy.de/heralhc/proceedings/heralhc-all.pdf.

[2] J. Collins, Phys. Rev. D 57 (1998) 3051 [Erratum-ibid. D 61 (2000) 019902]

[3] H1 Coll., H1prelim-10-014, H1prelim-10-012, H1prelim-10011

[4] ZEUS Coll., S. Chekanov et al., Nucl. Phys., B 816 (2009) 1

[5] ZEUS Coll., S. Chekanov et al., Nucl. Phys., B 831, (2010) 1

[6] ZEUS Coll., S. Chekanov, et al., Eur. Phys. J. C 52 (2007) 813.

[7] H1 Coll., H1prelim-10-017, H1prelim-09-011

[8] H1 Coll., A. Aktas et al., Eur. Phys. J. C 48, 715 (2006)

[9] ZEUS Coll., S. Chekanov, et al., Nucl. Phys. B 672 (2003) 3.

[10] ZEUS Coll., S. Chekanov, et al., Eur. Phys. J. C 55 (2008) 177. 